\title{
QUANTITATIVE ANALYSIS OF BANDED STRUCTURES IN DUAL-PHASE STEELS
}

\author{
Benoit Krebs $^{1}$, Alain Hazotte ${ }^{1}$, Lionel Germain ${ }^{1}$ And Mohamed Gouné2 \\ ${ }^{1}$ Laboratoire d'Etude des Textures et Application aux Matériaux (LETAM) FRE CNRS/UPVM 3143, Université \\ Paul-Verlaine, 57012 Metz cedex, France; ${ }^{2}$ ArcelorMittal Research SA, R\&D Automotive Products, Voie \\ Romaine - BP30320, 57283 Maizières-lès-Metz, France \\ e-mail: alain.hazotte@univ-metz.fr; lionel.germain@univ-metz.fr; mohamed.goune@arcelormittal.com \\ (Accepted April 28, 2010)
}

\begin{abstract}
Dual-Phase (DP) steels are composed of martensite islands dispersed in a ductile ferrite matrix, which provides a good balance between strength and ductility. Current processing conditions (continuous casting followed by hot and cold rolling) generate 'banded structures' i.e., irregular, parallel and alternating bands of ferrite and martensite, which are detrimental to mechanical properties and especially for in-use properties. We present an original and simple method to quantify the intensity and wavelength of these bands. This method, based on the analysis of covariance function of binary images, is firstly tested on model images. It is compared with ASTM E-1268 standard and appears to be more robust. Then it is applied on real DP steel microstructures and proves to be sufficiently sensitive to discriminate samples resulting from different thermo-mechanical routes.
\end{abstract}

Keywords: banded structure, covariance function, quantitative microscopy, steel.

\section{INTRODUCTION}

One of the major criteria in the development of advanced materials to meet the latest automotive fuel efficiency standards are mechanical properties improvements associated with high level of formability. Dual-Phase (DP) steels composed of martensite islands ( $\alpha$, phase in dark in Fig. 1) dispersed in a ductile ferrite matrix ( $\alpha$ phase in white in Fig. 1) were developed to provide a good balance between strength and ductility. In order to reach this goal, it is of prime necessity to control their final microstructure, in particular phase volume fraction, carbon composition and banded structure.

'Banded structure' or 'microstructural banding' are the terms used to qualify a microstructure consisting of parallel and alternating bands of ferrite and martensite (Fig. 1). A lot of publications (Bastien, 1957; Grossterlinden et al., 1992; Thompson and Howell, 1992) have been dedicated to the mechanisms of its formation. Only a few works have been published on quantitative characterization of microstructural banding through image analysis, although a lot of companies probably developed their own specific methods. ASTM E1268 standard (ASTM, 1988) was fixed to quantify microstructures presenting either banded structures or elongated second phase components. This method is based on counting the number of particle interceptions (or intersections) per unit length of straight lines parallel and perpendicular to the elongation direction. Two parameters are usually calculated to characterize microstructural banding: AI the anisotropy index defined as the ratio between the number of interceptions perpendicular and parallel to the deformation direction; $S B$ the mean center-tocenter spacing of the bands as the test line length divided by the number of interceptions perpendicular to the deformation direction. Since it is based on interception measurements, this method is very sensitive to sample preparation, especially on the presence of more or less boundaries between grains of the same phase (Hetzner, 1996; Krebs et al., 2010).

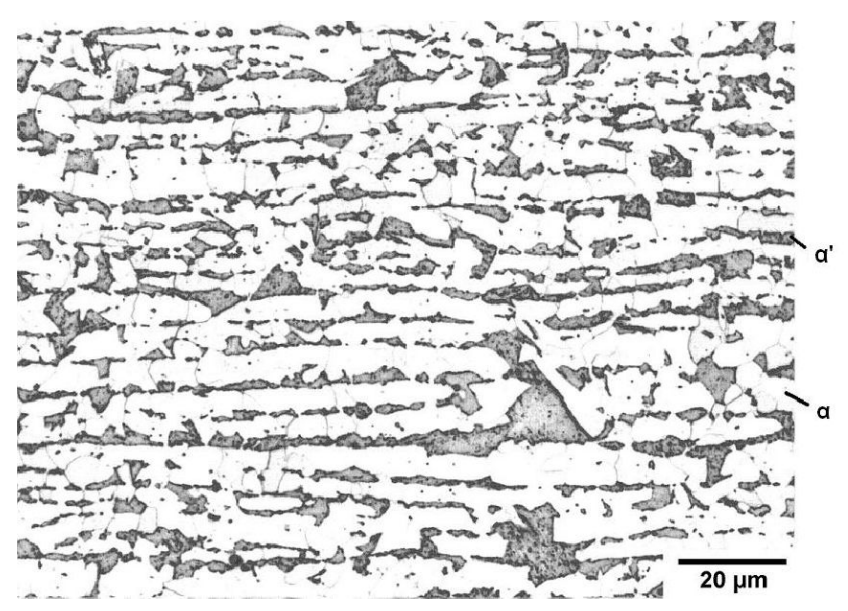

Fig. 1. Micrography of a banded structure of DualPhase steel; ferrite $(\alpha)$ appears in white, martensite $\left(\alpha^{\prime}\right)$ in dark. 
This paper present a novel and simple-toimplement method based on the automated analysis of the covariance function of binary images. This method is firstly validated on model images representative of banded microstructures, in order to evaluate its sensitivity and robustness. Then, the method is adapted and applied to discriminate banded DP microstructures resulting from three different heat treatment routes.

\section{QUANTITATIVE ANALYSIS METHOD}

\section{PRINCIPLE}

The covariance function has been introduced in the 60 's by G. Matheron as a simple but effective tool to describe the morphology of compact sets. More details on this function and on its applications can be found in references (Matheron, 1967; 1975; Serra, 1982; Stoyan et al., 1987; Coster and Chermant, 1989; Jeulin, 1997).

For the present purpose, the covariance can be introduced in a simple way: $X$ being a given compact set (phase) and $\vec{h}$ a vector (modulus $|h|$ and direction $\beta$ ) the covariance $C(X, \vec{h})$ is the probability that both extremities of vector $\vec{h}$ belong to $X$, whatever the position of $\vec{h}$ in the set. If $|h|=0$ this probability is equal to the volume fraction of $X$, i.e., $C(X, \overrightarrow{0})=V_{V}(X)$. If $|h|$ is very large, the probabilities for both extremities to belong to $X$ are independent and equal to $V_{V}(X)$, leading to $C(X, \vec{\infty})=V_{V}^{2}(X)$. Therefore, in the lack of any periodicity, the evolution of $C(X, \vec{h})$ as a function of $|h|$ (hereafter denoted as 'covariogram') shows a continuous asymptotic decrease from $V_{V}(X)$ to $V_{V}^{2}(X)$. The range of the covariogram - defined as the critical modulus $|h|$ over which $C(X, \vec{h})$ is 'sufficiently' close to the asymptote (using a given criterion) - is the distance over which two points in the microstructure can be considered as independent (non-correlated). Obviously, in the case of anisotropic microstructures, the shape of the covariogram will also depend on $\beta$. In addition, any periodicity in the compact set will result in oscillations of the covariogram before reaching the asymptote. The distance between oscillations and the magnitude of maxima are related to the wavelength, to the variability and to the intensity of the periodicity. The present work takes advantage of this particularity.

When dealing with binary digitized images, $\vec{h}$ is restricted to discrete vectors and $C(X, \vec{h})$ can be estimated through a very simple procedure: the initial image of $X$ set, $I(X)$, is translated by $\vec{h}$, giving a new image $I^{T \vec{h}} ; C(X, \vec{h})$ is measured as the area fraction of the intersection of these two images, $I(X) \cap I^{T \vec{h}}$. Obviously, the area fraction measurement has to be restricted to the effective surface of the overlap. The covariogram of $X$ set is obtained through successive translations with increasing $|h|$ modulus.

The parameters used to quantify the band structures in the following are schematically defined on Fig. 2 with the covariogram realized on a model microstructure. The mean distance between bands is estimated by the modulus of the first maximum of oscillations, noted $H M$ hereafter. Their intensity is quantified by the difference between $C(X, \vec{h})$ values measured at the first maximum $\left(C_{1 \text { Max }}\right)$ and first minimum $\left(C_{1 M i n}\right)$ of the covariogram. In the following, this difference $\left(C_{1 \text { Max }}-C_{1 \text { Min }}\right)$ is noted $B I$ (for Band Index). For randomly distributed grains of the analyzed phase, BI becomes very small but not null. In that case, HM is representative to the mean distance between grains. The slope at the origin of the covariogram, noted $C^{\prime}(X, \overrightarrow{0})$, or $C^{\prime} 0$, will also be used as an additional parameter sensitive to the grain size and the complexity of the microstructure. Its signification will be discussed in more details below. In the present work, this slope was estimated using a linear least square interpolation applied on the five first points of the covariogram as proposed by Coster and Chermant (1989). These three parameters are determined automatically during the covariogram acquisition. Unlike the ASTM method which measures individual intercepts, this approach is based on global surface measurements which prevents border effects.

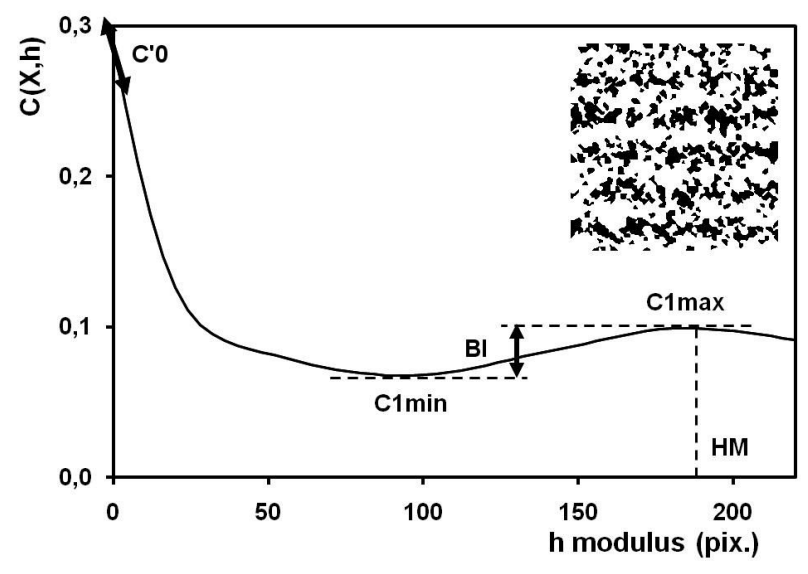

Fig. 2. Illustration of the parameters measured with covariogram method on a model microstructure.

\section{VALIDATION}

Validation of the method is described in more details elsewhere (Krebs etal., 2010). Only its 
principle and main conclusions are given here. Model images of banded microstructures have been created with Voronoi tesselations, in order to test the efficiency of the analysis procedures without interfering with the problems of experimental part. The image analysis software APHELION (copyright ADCIS S.A. and A.A. Imaging) was used for this purpose, as well as for the development of the different algorithms described hereafter. Each microstructure was generated with 2000 nuclei in $1000 \times 1000$ pix. $^{2}$ image. $P\left(y_{i}\right)$ the probability to get martensite or ferrite, was defined as following:

$$
P\left(y_{i}\right)=V_{V}+\frac{\Delta V_{V}}{2} \sin \frac{2 \pi}{\lambda \pm \varepsilon}\left(y_{i}-y_{r}\right),
$$

where $V_{V}$ is the mean martensite volume fraction, $\Delta V_{V}$ is the banding magnitude and $\lambda$ is the band wavelength; $y_{r}$ is a random number ranging between 0 and $\lambda$, which allows to vary the vertical position of rich and poor bands from one image to another and $\varepsilon$ follows a Gaussian distribution of average 0 and standard deviation $\Delta \lambda$ and varies the interspacing from one band to the next.

The covariogram method was compared to the automated version of ASTM E-1268 presented in 1996 (Hetzner, 1996). The method based on the covariance function appears much more robust with regard to the sample preparation (Krebs et al., 2010). Fig. 3 reports the evolution of $B I$ and $H M$ parameters, as a function of the microstructure parameters. It can be seen that $B I$ evolves linearly with $\Delta V_{V}^{2}$, whatever the value of $V_{V}$. $H M$ values are close to the real band wavelength while these parameters measured with the ASTM method are unrealistic (Krebs et al., 2010).

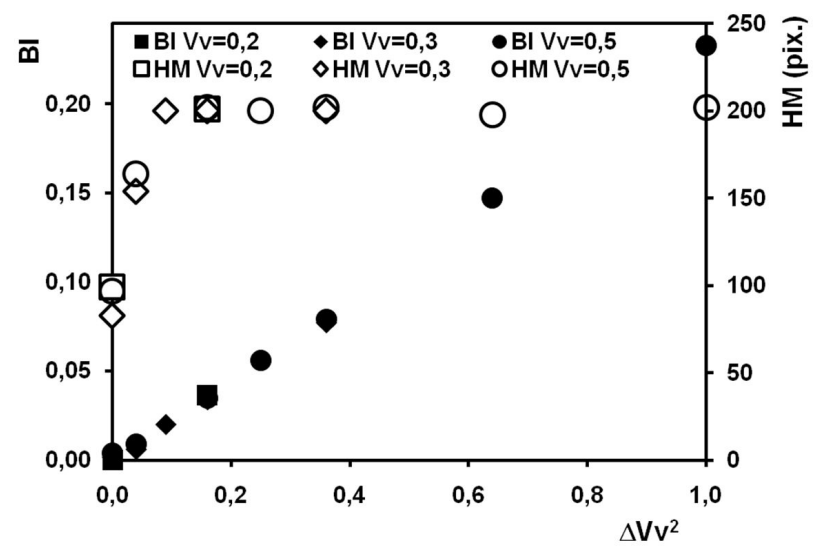

Fig. 3. Evolution of BI and $H M$ as a function of banding magnitude, $\Delta V_{V}$, for different mean martensite volume fractions ( $\lambda=200$ pix. $)$.

\section{APPLICATION TO DUAL-PHASE STEELS}

\section{MATERIALS}

The microstructures tested were extracted from a wider set of experiments focusing on the mechanisms of solid phase transformation during continuous cooling of DP steels. The chemical composition of the Fe-C-Mn alloy used is reported in Table 1. Small samples were extracted from $1.2 \mathrm{~mm}$ thick sheets issued from a complex route involving continuous casting followed by hot and cold rolling. For one of these sheets, an additional homogenization heat treatment was performed to smooth the segregation of the Mn element.

Table 1. DP steel composition.

\begin{tabular}{lcccccc}
\hline Element & $\mathrm{C}$ & $\mathrm{Mn}$ & $\mathrm{Si}$ & $\mathrm{P}$ & $\mathrm{S}$ & $\mathrm{Fe}$ \\
\hline Wt pct & 0.15 & 1.48 & 0.013 & 0.01 & $27 \mathrm{ppm}$ & 98.34 \\
\hline
\end{tabular}

Then, the samples were given specific heat treatments schematized in Fig. 4, in order to obtain different types of DP structures. For the present study, two cooling rates, $\rho_{c}$, from $870^{\circ} \mathrm{C}$ down to $650^{\circ} \mathrm{C}$ were selected: a 'standard' (slow) cooling rate (SC) and a rapid one (RC). Then, several holding times at $650^{\circ} \mathrm{C}, \mathrm{t}_{\alpha}$, were applied to initiate various amounts of ferrite before quenching.

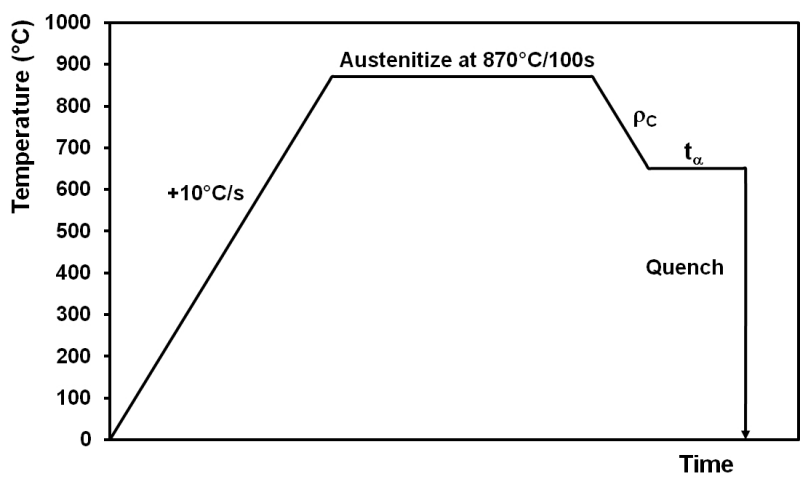

Fig. 4. Temperature cycle used to obtain a DP microstructure.

Table 2 summarizes the heat-treatment parameters of all the samples analyzed in the present work, together with their corresponding denominations. Heat-treated samples were cut perpendicular to the sheet plane and parallel to the rolling direction, then polished by conventional mechanical grinding. DP microstructures were revealed by Vilella etching (4 $\mathrm{g}$ picric acid, $1 \mathrm{ml} \mathrm{HCl}, 100 \mathrm{ml}$ ethanol) at 
room temperature during 4 seconds. Images were acquired with an optical microscope, Olympus BX61, equipped with a high resolution digital camera (2080 $\times$ 1544 pix. $^{2}$ ). Phase amounts were automatically measured over at least 20 images per sample using the conventional method, i.e., 'manual' grey-level thresholding of martensite phase then pixel counting. Last column of Table 2 also reports the measured volume fraction of martensite for every heat-treated sample.

Covariograms of the martensite phase were measured perpendicular to the rolling direction, i.e., perpendicular to the bands, with $|h|$ increasing step by step (step size ranging from 1 to 4 pixels, depending on the image size and precision required). At least ten images per - model or real - microstructure were analyzed to obtain an averaged curve.

In the microstructures showed in Fig. 1, the bands of martensite are globally horizontal, but they slightly wave along the width of the pictures. These horizontal fluctuations tend to smooth the covariograms if applied on a too large region. Thus, in the case of real DP microstructures, we chose to perform the covariogram algorithm on pictures narrower than the mean period of the horizontal waves. This period was estimated at about $90 \mu \mathrm{m}$ by measuring the range of covariograms in the horizontal direction. Therefore, the pictures from the light microscope $\left(144 \times 106 \mu \mathrm{m}^{2}\right.$ in size $)$ were systematically divided into eight sub-pictures (i.e., $36 \times 53 \mu \mathrm{m}^{2}$ ) before processing. Consequently, at least 160 sub-pictures were analyzed for each sample; the $B I, H M$ and $C^{\prime} 0$ results reported hereafter are mean values calculated over the whole sampling.

Table 2. Heat treatments applied in the present work, corresponding samples names and measured martensite volume fractions $V_{V}\left(\alpha^{\prime}\right)$.

\begin{tabular}{|c|c|c|c|c|}
\hline Material & $\begin{array}{c}\rho_{c} \\
\left({ }^{\circ} \mathrm{C} / s\right)\end{array}$ & $\begin{array}{l}\mathrm{t}_{\alpha} \\
(\mathrm{s})\end{array}$ & $\begin{array}{c}\text { Sample } \\
\text { name }\end{array}$ & $V_{V}\left(\alpha^{\prime}\right)(\%)$ \\
\hline \multirow{10}{*}{ Banded } & \multirow{6}{*}{-10} & 0 & SC_0 & $41.1 \pm 1.2$ \\
\hline & & 5 & SC_5 & $38.1 \pm 0.9$ \\
\hline & & 10 & SC_10 & $35.4 \pm 0.6$ \\
\hline & & 20 & SC_20 & $33.2 \pm 0.7$ \\
\hline & & 30 & SC_30 & $30.5 \pm 0.6$ \\
\hline & & 60 & SC_60 & $28.7 \pm 0.4$ \\
\hline & \multirow{4}{*}{-100} & 5 & RC_5 & $39.3 \pm 0.7$ \\
\hline & & 10 & $\mathrm{RC}_{-} 10$ & $36 \pm 0.7$ \\
\hline & & 15 & RC_15 & $35.2 \pm 0.5$ \\
\hline & & 60 & RC_60 & $31.9 \pm 0.5$ \\
\hline \multirow{3}{*}{ Homogeneous } & \multirow{3}{*}{-10} & 0 & H_0 & $30.3 \pm 1.4$ \\
\hline & & 5 & H_5 & $26.8 \pm 1.3$ \\
\hline & & 10 & H_10 & $25.6 \pm 1.6$ \\
\hline
\end{tabular}

\section{RESULTS AND DISCUSSION}

Some values of $B I$, measured for different microstructures (modeled or measured) presenting almost the same martensite volume fraction (about $30 \%$ ), are reported in Fig. 5. For the same microstructure parameters $\left(V_{V}=0.3 ; \quad \Delta V_{V}=0.6\right) \quad B I$ is divided by more than two between a regular microstructure (BV_SIN) and a microstructure with wavelength variation $\Delta \lambda$ (BV_SIN $\Delta \lambda=100$ pix.). On real microstructures, the band index is higher in the case of standard cooling (SC_30) than for the rapid one (RC_60). As expected $B I$ is very small for homogeneous microstructures (H_0 and HV), which confirm that they present no banding.

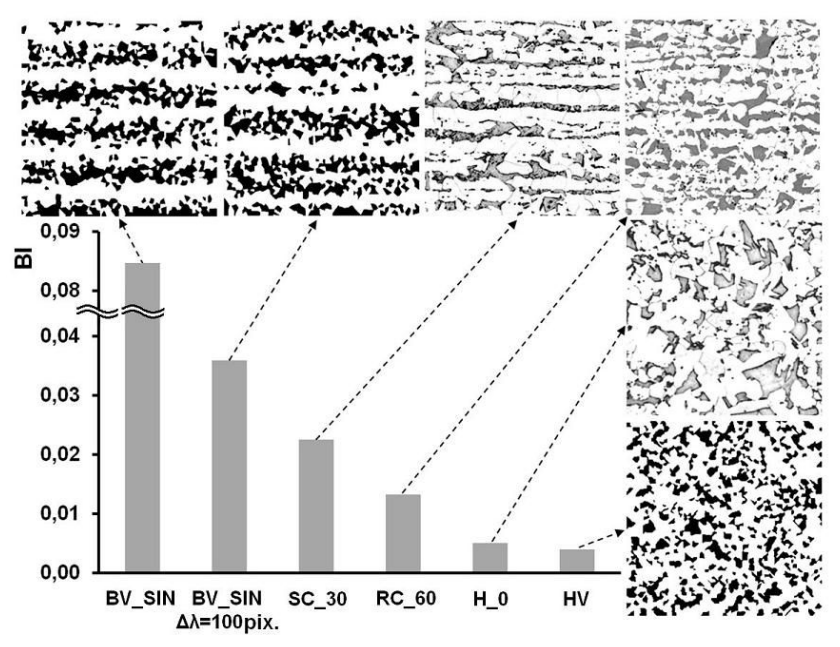

Fig. 5. Values of BI for (from left to right) a banded Voronoi microstructure $\left(V_{V}=0.3, \Delta V_{V}=0.6\right)$; the same microstructure with $\Delta \lambda=100$ pix.; $S C \_30$ microstructure; $R C \_60$ microstructure; $H_{-} 0$ microstructure and a homogeneous Voronoi microstructure $\left(V_{V}=0.3\right)$.

Fig. 6 shows the average values of $B I, H M$ and $C^{\prime} 0$ plotted as functions of martensite volume fraction, for the slow (SC) and rapid cooled (RC) banded microstructures, as well as for the homogeneous $(\mathrm{H})$ microstructures. The $95 \%$ confidence interval was computed as $\pm \frac{2 \sigma}{\sqrt{n}}$, with $\sigma$ the standard deviation calculated on $n$ sub-pictures. $B I$ appears as a relevant parameter to discriminate microstructures as a function of their banding intensity. The values of $B I$ are very distinct from one structure to another, while being relatively independent on the martensite volume fraction. SC and RC microstructures are well discriminated as the values of $B I$ for SC are almost twice those for RC, except for the value measured for $41.1 \%$ of martensite. The decrease of $B I$ at high volume fractions of martensite result from the fact that 
martensite grains connect from one band to the next, leading to a less pronounced banded structure.

The wavelength of the bands is well estimated by $H M$. For the banded RC and SC materials - which have been submitted to a similar thermo-mechanical route except the last transformation cooling step $-H M$ is relatively constant within the range $5 \pm 0.2 \mu \mathrm{m}$. For the homogeneous material - which was submitted to a long homogenising heat treatment resulting in a coarser microstructure $-H M$ is found slightly higher, around $6.5 \pm 0.25 \mu \mathrm{m}$. Although $H M$ values of homogeneous samples are associated with a very low banding index, they are rather reproducible and give an estimation of the mean (isotropic) distance between two grains of martensite.

The larger size of martensite grains in the homogeneous samples is confirmed by the higher levels of $C^{\prime} 0$ values measured in comparison with those of the banded ones (Fig. 6). $\left|C^{\prime} 0\right|$ is also found to systematically increase with increasing martensite volume fraction, whatever the type of microstructure. The following relationship between $\left|C^{\prime} 0\right|$ and $S B$ (the mean center-to-center spacing of the bands calculated using the ASTM method) can be easily showed (Krebs, 2009):

$$
\left|C^{\prime} 0\right| \approx \frac{1}{S B}
$$

This relation shows that $S B$ depends also on the volume fraction. The evaluation of the distance between bands with the covariogram method appears more physical and robust than its evaluation based on ASTM method. In addition, the rate of this increase appears to be higher for the rapidly-cooled samples as compared to the slowly-cooled ones. This evolution is more delicate to analyze. Indeed, $\left|C^{\prime} 0\right|$ is expected to rise with increasing length of the interface perimeter projected in the direction of $\vec{h}$ (Serra, 1982; Coster and Chermant, 1989). This means that it will increase if the phase components are refined at constant shape, but also if their shape becomes more corrugated at constant size.

In the case of DP steels, martensite phase $\alpha^{\prime}$ results from a complex transformation route including size, shape and topology changes. $\alpha^{\prime}$ phase is the trace of the high-temperature austenite phase $\gamma$ nontransformed during the $\gamma \rightarrow \alpha$ transformation. Thus, higher $\alpha^{\prime}$ amounts are associated with earlier stages of transformation - i.e., coarser blocks of embedding austenite with complex shapes - whereas lower amounts corresponds to finer but more compact grains.

The global evolution observed for $C^{\prime} 0$ vs $V_{V}\left(\alpha^{\prime}\right)$ suggests that $C^{\prime} 0$ could be sensitive to the shape/topology changes as well as to the mean size variations for the type of microstructures tested. Moreover, $C^{\prime} 0$ variation between $\mathrm{SC}$ and $\mathrm{RC}$ samples could be the trace of a difference in $\gamma \rightarrow \alpha$ transformation mechanisms. Indeed, it is suspected that increasing the cooling rate would result in a transition from a mechanism involving isotropic growth of compact ferrite grains to a mechanism characterized by the development of long acicular $\alpha$ grains.
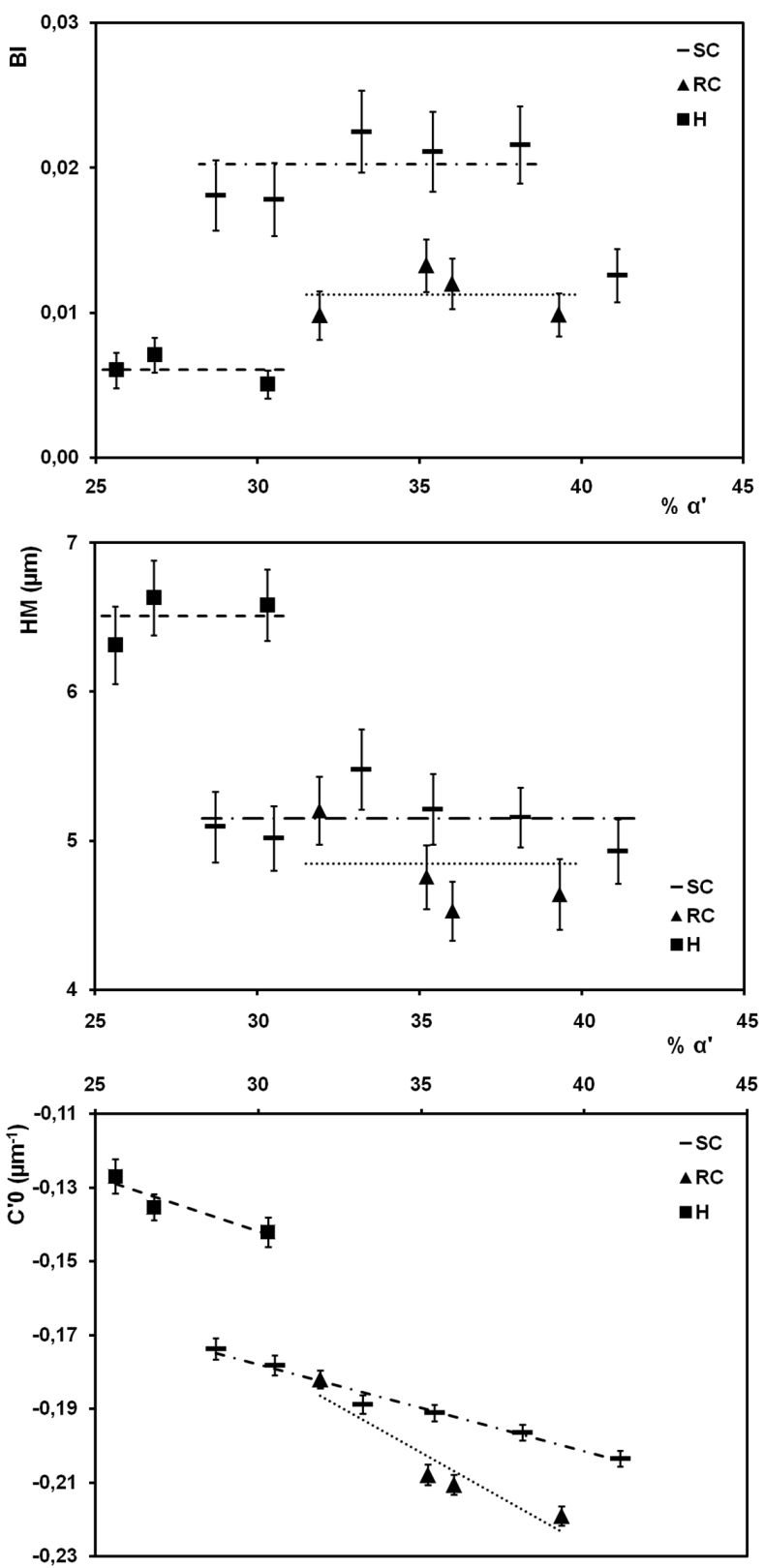

Fig. 6. Evolution of BI, HM and $C^{\prime} 0$ as functions of martensite content for the three types of microstructures. 


\section{CONCLUSION}

We proposed a novel and simple-to-implement method to quantify the periodicity and intensity of banded structures in materials. This robust and sensitive method based on the automated analysis of the covariance function of binary images was tested either on model images or on real microstructures of Dual-Phase (DP) steels. It is particularly efficient to quantify banded structures with relatively low amount of segregated phase, as in the case of DP steels. In the present work, the measured covariograms were reduced to only three parameters: the banding index $B I$, the modulus at first maximum $H M$ and the slope at the origin $C^{\prime} 0 . B I$ is very sensitive to compare microstructures inherited from different process. $H M$ gives a reliable and robust measure of interspaces between bands. $C^{\prime} 0$ depends in a complex way discussed in this paper on the fineness and morphology of the microstructure. This last one can be also helpful to describe transformation mechanism.

\section{ACKNOWLEDGEMENTS}

This work was financially supported by ArcelorMittal Company, by the 'Centre National de la Recherche Scientifique' (French research council) and by the 'Région Lorraine'.

\section{REFERENCES}

ASTM International (1988). Standard Practice for Assessing the Degree of Banding or Orientation of Microstructures. E 1268-01 (Reapproved 2007).
Bastien PG (1957). The mechanism formation of banded structures. J Iron Steel Inst 193:281-91.

Coster M, Chermant JL (1989). Précis d'analyse d'images. Paris : Les Presses du CNRS.

Grossterlinden R, Kawalla R, Lotter U, Pircher H (1992). Formation of pearlitic banded structures in ferriticpearlitic steels. Steel Res 63:331.

Hetzner DW (1996). Quantitative assessment of banding by automatic image analysis. Microstruc Sci 24:211-25.

Jeulin D (1997). Advances in theory and applications of random sets. Singapore: World Scientific.

Krebs B (2009). Ph.D. thesis. Metz: Université PaulVerlaine.

Krebs B, Germain L, Hazotte A, Gouné M (2010). Banded structures in dual-phase steels - A novel characterization method. Int J Mater Res 2011:200-12.

Matheron G (1967). Eléments pour une théorie des milieux poreux. Paris: Masson.

Matheron G (1975). Random sets and integral geometry. New York: Wiley.

Serra J (1982). Image analysis and mathematical morphology. London: Academic Press.

Stoyan D, Kendall WS, Mecke J (1987). Stochastic geometry and its applications. Chichester: Wiley.

Thompson SW, Howell PR (1992). Factors influencing ferrite/pearlite banding and origin of large pearlite nodules in a hypoeutectoid plate steel. Mater Sci Technol 8:777-84. 